\title{
Chronic Invasive Fungal Rhinosinusitis with Atypical Clinical Presentation in an Immunocompromised Patient
}

This article was published in the following Dove Press journal:

Infection and Drug Resistance

\author{
Yuka Kawaji-Kanayama (D) \\ Ayako Nishimura ${ }^{2}$ \\ Makoto Yasuda $\mathbb{D}^{3}$ \\ Emiko Sakiyama (1D ${ }^{1,4}$ \\ Yuji Shimura (D) \\ Taku Tsukamoto' \\ Tsutomu Kobayashi (ID) \\ Shinsuke Mizutani' \\ Shota Okamoto ${ }^{3}$ \\ Gaku Ohmura ${ }^{3}$ \\ Shigeru Hirano ${ }^{3}$ \\ Eiichi Konishi $\mathbb{D}^{2}$ \\ Kazutoshi Shibuya ${ }^{5}$ \\ Junya Kuroda (D) ${ }^{\prime}$
}

'Division of Hematology and Oncology, Department of Medicine, Kyoto

Prefectural University of Medicine, Kyoto, Japan; ${ }^{2}$ Division of Surgical Pathology,

Department of Pathology, Kyoto

Prefectural University of Medicine, Kyoto, Japan; ${ }^{3}$ Department of Otolaryngology-

Head and Neck Surgery, Kyoto

Prefectural University of Medicine, Kyoto, Japan; ${ }^{4}$ Department of Hematology,

Matsushita Memorial Hospital, Moriguchi, Japan; ${ }^{5}$ Department of Surgical Pathology,

Toho University Graduate School of

Medicine, Tokyo, Japan

Correspondence: Junya Kuroda Division of Hematology and Oncology, Department of Medicine, Kyoto

Prefectural University of Medicine, 465

Kajii-Cho, Kamigyo-Ku, Kyoto 602-8566,

Japan

Tel +8I 7525 I 5740

Fax $+817525 I 5743$

Email junkuro@koto.kpu-m.ac.jp

\begin{abstract}
Invasive fungal rhinosinusitis (FRS) is a rare but intractable infectious disease of the sinonasal region with destructive direct infiltration into surrounding tissues, such as the bone, orbit and brain, and potential dissemination to systemic organs. Symptomatic assessments and imaging are frequently not sufficiently diagnostic, and histopathological examination is essential for definite diagnosis of FRS. We herein report a case of chronic invasive FRS (CIFRS) in a 58-year-old Japanese male with end-stage diabetic nephropathy that required maintenance dialysis after graft rejection of living kidney transplantation. His initial main clinical presentation was sinus gangrene, which gradually progressed from the paranasal sinus to the nasal septum and oral palate, but not towards the intracranial or orbital region, for two months. The patient was first strongly suspected to have extranodal natural killer/T cell lymphoma (ENKTL), nasal type, a subtype of malignant lymphoma, based on the macroscopic appearance of the gangrene, expansion pattern and high serum soluble interleukin-2 level; however, repeated biopsies and eventual resection led to diagnosis of CIFRS due to Aspergillus niger and Mucor. The disease was improved by surgical resection in combination with antifungal pharmacologic treatment with liposomal amphotericin B and voriconazole. CIFRS typically occurs in immunocompetent patients and shows intracranial progression, but this case shows that atypical CIFRS with an uncommon expansion pattern can occur in an immunodeficient patient.
\end{abstract}

Keywords: chronic invasive fungal rhinosinusitis, diabetes mellitus, maintenance dialysis, extranodal natural killer/T cell lymphoma, nasal type

\section{Introduction}

Fungal rhinosinusitis (FRS) presents with various clinical manifestations depending on the immune status, and the incidence has increased due to an increase in immunosuppressed patients with diabetes mellitus (DM) and cases with acquired immunodeficiency after chemotherapy or immunosuppressant therapy. ${ }^{1-3}$ Extramucosal non-invasive forms of FRS, including superficial sinonasal mycosis, allergic FRS and fungal ball, are common in daily practice, whereas invasive forms, such as chronic invasive FRS (CIFRS) and acute fulminant invasive FRS (AIFRS), are rare. The prognosis of invasive FRS is poor due to its aggressive disease manifestation with destructive invasion of surrounding tissues, especially intracranial and orbital lesions, and occasional dissemination to systemic organs. ${ }^{1-6}$

Since clinical symptoms, gross appearance and imaging findings are frequently similar to those of malignant tumors or granulomatous diseases, ${ }^{2,3,7}$ a histopathologic 
examination is required for the correct diagnosis and appropriate treatment choice for invasive FRS. ${ }^{8-10}$ However, diagnosis is not always straightforward, even with histopathologic assessments. Here, we report a case of clinically atypical CIFRS in an immunocompromised patient that expanded towards the nasal cavity and oral palate, but not to the intracranial region.

\section{Case Report}

A 58-year old Japanese male with type 2 DM and related end-stage chronic renal failure (CRF) was referred to our hospital for diagnosis and treatment of rhinosinusitis of undetermined etiology. The patient visited a local hospital complaining of headache, a depressed nasal bridge and nasal dryness with purulent rhinorrhea. He had a history of living renal transplantation for CRF. Removal of the grafted kidney had become necessary due to acute rejection one month before he became aware of sinonasal symptoms, and he had been treated by maintenance hemodialysis. Immunosuppressants started just after transplantation had already been discontinued by the day of his first visit.

At the onset, a computed tomography (CT) scan showed the partial fracture of septum and soft tissue density in the paranasal sinuses (Figure $1 \mathrm{~A}$ and $\mathrm{B}$ ). The patient was initially suspected to have sinusitis, but the paranasal pyogenic lesion gradually progressed to development of nasal septum perforation, crust formation and saddle nose. Eventually necrotic tissue penetrated through the hard palate to the oral cavity after one month of therapy, and the lesion was considered to be a hidden tumor of extranodal natural killer/T cell lymphoma (ENKTL), nasal type. A biopsy of the necrotic tissue from the nasal cavity and hard palate had been performed at the previous hospital, but no diagnostic specimen was obtained. In the absence of definite histopathologic findings, the patient was suspected to have ENKTL, nasal type based on the macroscopic appearance and laboratory data. He was admitted to our hospital for further examination and treatment at three months after onset of rhinosinusitis.

On admission, the patient had a deteriorated condition and his Eastern Cooperative Oncology Group performance status was grade 3. Endoscopic examination revealed the perforation of nasal septum, large amounts of crusts in the nasal cavity (Figure 2A), and necrotic tissue on the hard to soft palate (Figure 2B). In addition, rotten bone appeared, when the crusts were removed. Blood tests revealed a normal leukocyte count of $6.20 \times 10^{9} / \mathrm{L}$ (normal range:
3.30-8.60 $\times 10^{9} / \mathrm{L}$ ) comprising $71.0 \%$ neutrophils (normal range: $39.8-70.5 \%$ ) and $2.0 \%$ eosinophils (normal range:0.6-5.4\%). Serological examination identified elevation of blood urea nitrogen to $79.1 \mathrm{mg} / \mathrm{dL}$ (normal range: 8.0-20.0 $\mathrm{mg} / \mathrm{dL}$ ) and creatine to $5.87 \mathrm{mg} / \mathrm{dL}$ (normal range: $0.65-1.07 \mathrm{mg} / \mathrm{dL}$ ). Hemoglobin A1c (NGSP) was $5.9 \%$ (normal range: 4.9-6.0\%). Soluble interleukin-2 receptor (sIL-2R) was markedly elevated to $3280 \mathrm{U} / \mathrm{mL}$ (normal range: $157-474 \mathrm{U} / \mathrm{mL}$ ) and C-reactive protein was slightly elevated to $1.71 \mathrm{mg} / \mathrm{dL}$ (normal range: $<0.20 \mathrm{mg}$ / dL). $\beta$-D glucan was elevated to $18.1 \mathrm{pg} / \mathrm{mL}$ (normal: $<11.0 \mathrm{pg} / \mathrm{mL}$ ), while Aspergillus antigen, EBV-DNA and anti-neutrophil cytoplasmic antibody were negative. Nasal bacterial cultures failed to detect the pathogenic microorganism. Magnetic resonance imaging (MRI) showed destruction of the nasal structure with loss of the septum, and abnormal mucosal thickening and mucus retention in the sinuses, but no tumor was detected in the sinonasal region (Figure 3 ). The destructive area did not reach the intracranial region, despite expansion and penetration towards the oral cavity. Repeated conventional biopsies of small tissue samples from the surface of sinus gangrene using biopsy forceps showed the presence of necrotic tissue with numerous fungi, including Candida species and Mucor, and Actinomycetes, which extended deep below the mucosa (Figure $4 \mathrm{~A}$ and $\mathrm{B}$ ). However, it was difficult to conclude whether those microorganisms were truly pathogenic or merely contaminations, as the histologic finding lacked key-point features for the diagnosis of CIFRS, such as fungal invasion within the vessels or mucosal/vascular necrosis, perhaps due to massive tissue destruction by necrosis which caused the disappearance of findings for vascular invasion and mucosal/vascular necrosis. It was also difficult to make a definite differential diagnosis from malignant lymphoma, such as ENKTL, nasal type, due to the small tissue volume of the biopsied samples.

Accordingly, we tentatively diagnosed CIFRS and started antifungal treatment with liposomal amphotericin B (L-AMB), while careful attention was paid to possible emergence of ENKTL. However, the gangrene still continued to expand towards the soft palate despite treatment with L-AMB for two weeks, and, indeed, CT examination showed no major improvement of sinus abnormal lesion (Figure 1C and D). Therefore, we next performed endoscopic sinus surgery under general anesthesia with the aim of both removal of necrotic tissue and histopathologic examination. MRI after endoscopic surgery showed the 


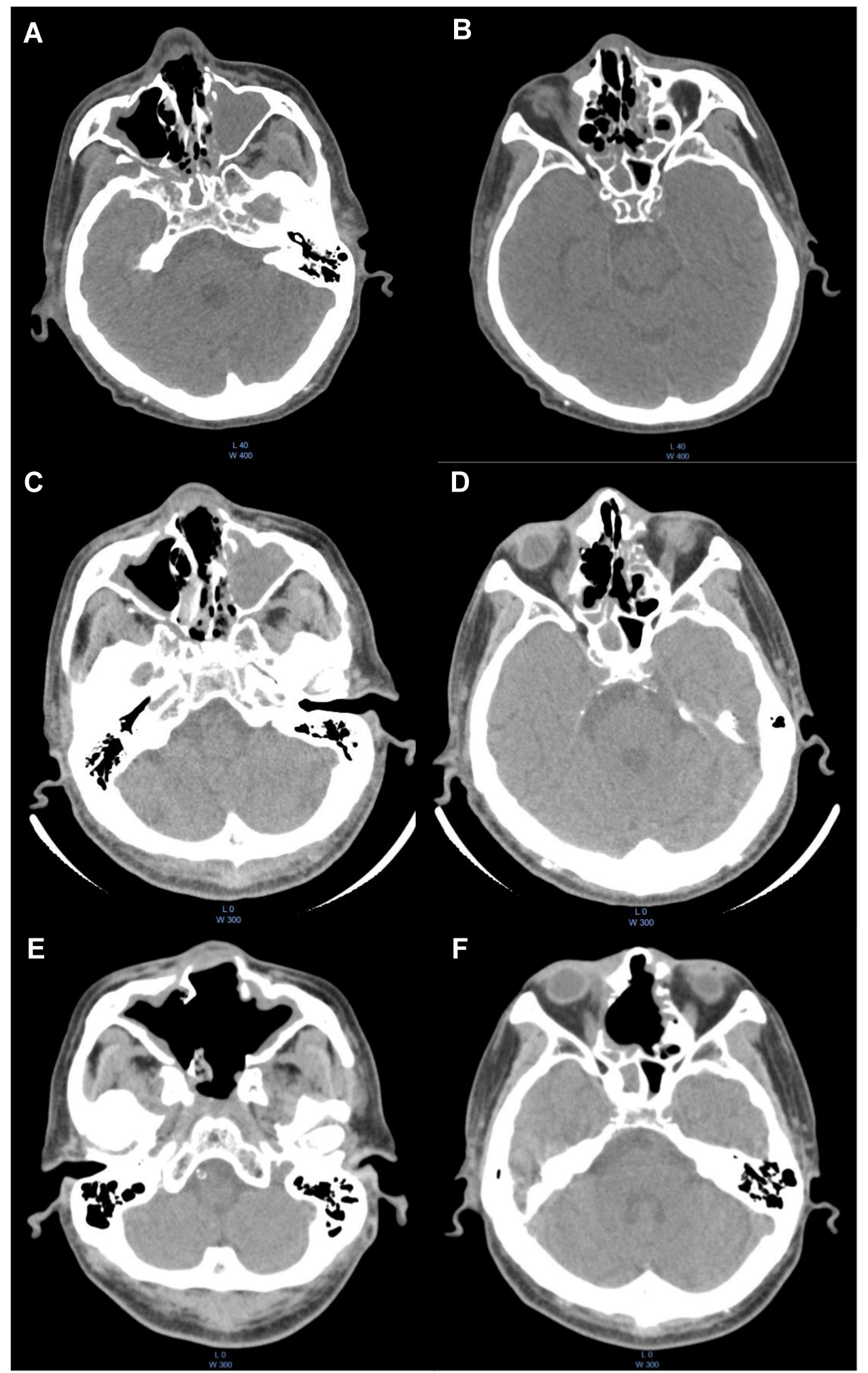

Figure I Computed tomography (CT) at the level of the maxillary sinus ( $\mathbf{A}$ and $\mathbf{C}$ and $\mathbf{E}$ ) and the ethmoid sinus (B and $\mathbf{D}$ and $\mathbf{F})$. (A and $\mathbf{B})$ The nasal septum fracture and soft tissue density in sinus cavity were observed at the onset, those were not remarkable changed just before endoscopic sinus surgery (C and $\mathbf{D})$. (E and $\mathbf{F})$ Follow-up $C T$ scan after endoscopic surgery (before additional surgery) showed the removal of fractured nasal septum and the improvement of mucosal thickening. 


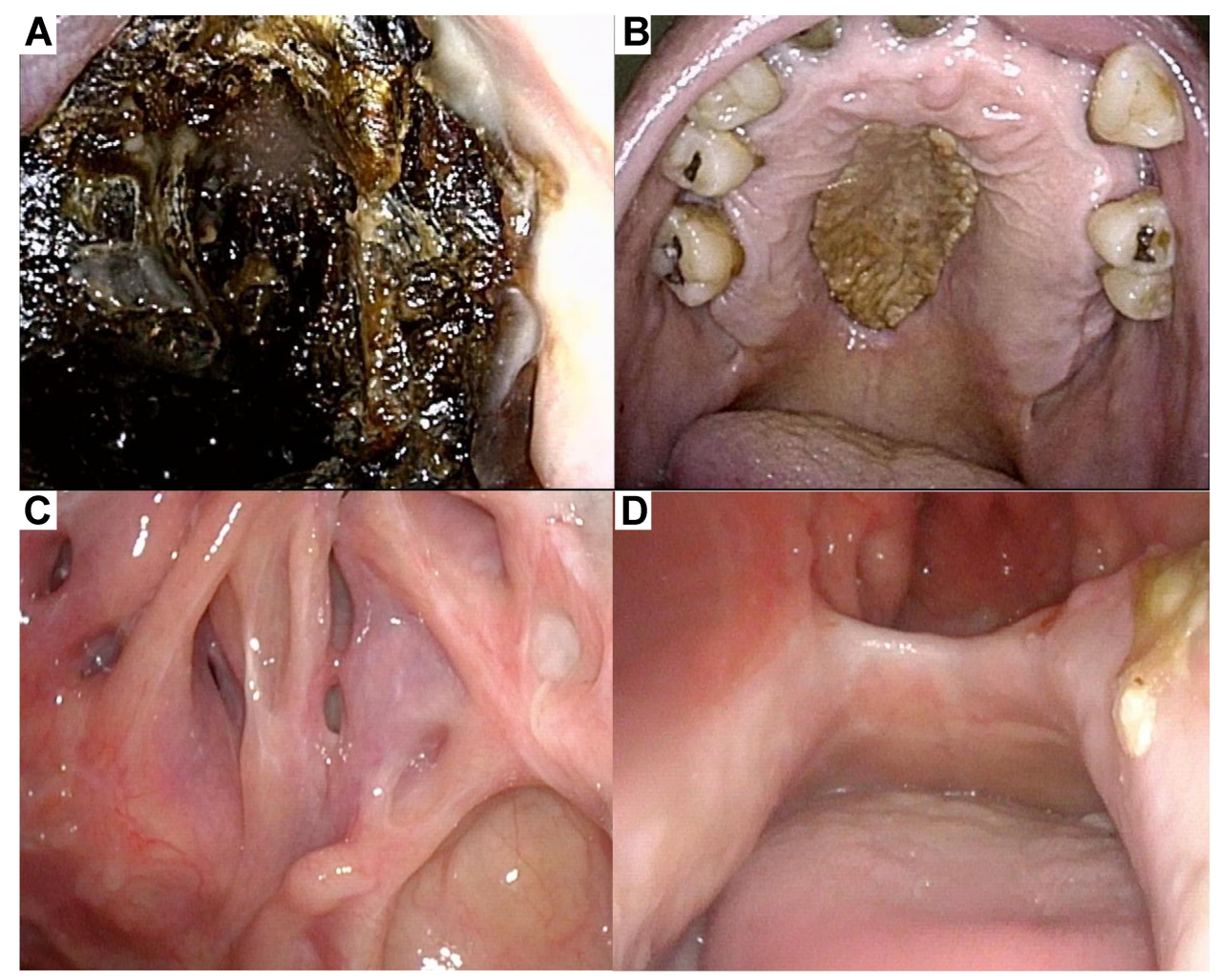

Figure 2 Endoscopic findings of progressive gangrene. (A) The destroyed nasal cavity was heavily crusted on admission. (B) Necrotic tissue on the oral palate at admission. (C) Sphenoid sinuses and (D) palate defect four months after additional necrotomy. Almost no necrotic tissue remained at either site.

removal of septum, but the residual soft tissue (Figure 5A and B). The resected specimen showed extensive infiltration of Aspergillus niger in the background of necrotic tissue, but again without evidence of tumor tissue (Figure 4C-E), based on which we made a final conclusive diagnosis of CIFRS, despite the absence of finding for fungal vessel invasion. Thereafter, the patient was treated with oral voriconazole and routine nasal cleaning as an outpatient, and his symptoms gradually improved. Followup CT scan (Figure 1E and F) and MRI (Figure 5C and D) showed no intracranial extension and the gradual improvement of the mucosal thickening, however, he unfortunately experienced a skin perforation of the nasal root after antifungal treatment for seven months, and an additional operation was performed to remove residual necrotic tissue on the palate and paranasal sinuses eight months after his first admission to our hospital. Presently, there is almost no necrotic tissue in his nasal cavity and paranasal sinus (Figure 2C and D) and MRI shows only slight residual mucosal thickening (Figure $5 \mathrm{E}$ and $\mathrm{F}$ ).

\section{Discussion}

FRS is classified into invasive and non-invasive forms with and without fungal infiltration into mucosa. ${ }^{1-6,11}$
Invasive FRS is further subcategorized into AIFRS, which normally progresses within four weeks, and CIFRS, which progresses over more than four weeks. ${ }^{2,4}$ Aspergillus is the most frequent pathogen for FRS and Mucor is also commonly causative, regardless of the FRS subtype. ${ }^{1-6}$ Malignant diseases such as lymphomas or epithelial carcinomas, and non-malignant diseases, including granulomatosis with polyangiitis (ie Wegener granulomatosis) and FRS, can destroy the normal nasal and sinus structure and cause gangrene. Among these diseases, a distinct pathological and clinical characteristic of invasive FRS is related to the biological characteristics of Aspergillus and Mucor; that is, their preference for intracranial invasion due to the vascular affinity of fungi. This unique characteristic is also associated with the increased incidence of systemic dissemination leading to diseases such as meningitis, encephalitis, abscesses, infectious aneurysms, cerebral infarction, and cerebral hemorrhage in invasive FRS. ${ }^{12-14}$

Despite its unique features, definite diagnosis of invasive FRS is frequently difficult in daily clinical practice because the gross appearance and symptoms of invasive FRS due to destruction of the sinus and nasal cavity are frequently similar to those in the other diseases mentioned 


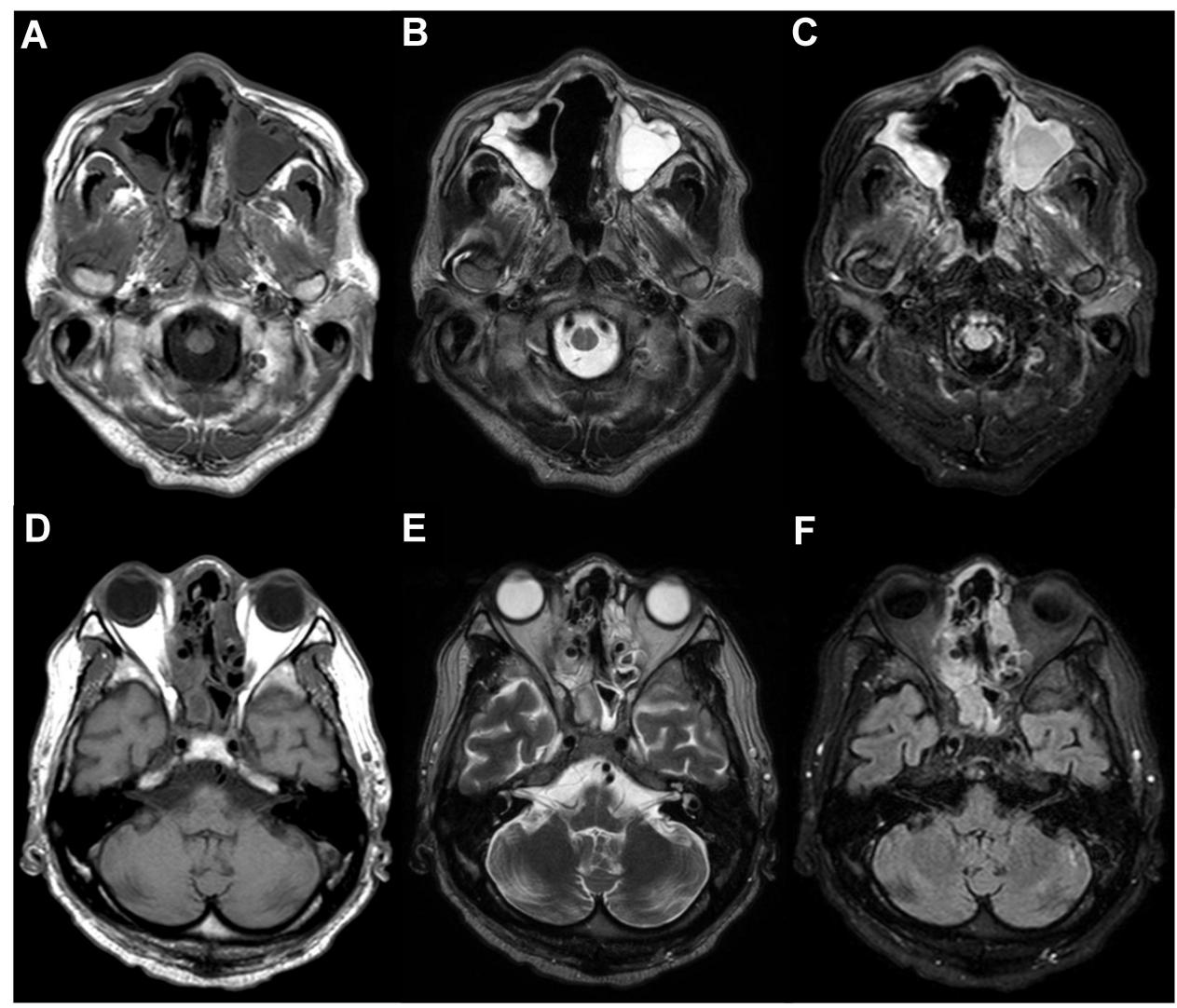

Figure 3 Magnetic resonance imaging (MRI) on admission. Horizontal views of (A and D) TI-weighted, (B and E) T2-weighted, and (C and F) FLAIR images at the level of $(\mathbf{A}-\mathbf{C})$ the maxillary sinus and $(\mathbf{D}-\mathbf{F})$ the eyeballs. The nasal septum was disrupted $(\mathbf{A}-\mathbf{C})$ and structural deformation with mucosal thickening and fluid retention were observed in the maxillary, ethmoid and sphenoid sinuses (A-F).

above. ${ }^{2,3,7}$ The sensitivity of culture tests for pathogenic fungi is insufficient ${ }^{5,15}$ and the diagnostic powers of imaging modalities are also limited for invasive FRS, although septal enhancement or loss of contrast enhancement on MRI is informative in the differentiation of invasive FRS and malignant tumors. ${ }^{16,17}$ Therefore, histopathological assessment is required for diagnosis of FRS. ${ }^{5,10,15,18}$ However, even in the absence of tumor cells in a biopsied specimen, it is not always easy to exclude the possibility of a false-negative test for the presence of a cancerous disease that has developed around the necrotic lesion, especially in a case in which only a small biopsy specimen of the gangrenous lesion is available for assessment.

The present case was finally diagnosed as CIFRS based on histopathologic examination of a relatively large resected specimen and the time needed for progression of over two months. However, the diagnosis was confusing for several reasons at initial presentation. First, the expansion pattern of the gangrene was uncommon for invasive FRS, as it only extended from the sinus to nasal septum and nasal cavity to oral palate, but not towards the intracranial or orbital region. The clinical appearance, including the expansion pattern, imaging tests and high serum sIL-2R level all initially suggested malignant lymphoma, especially ENKTL, nasal type, which is more common in Asian patients. This is a relatively rare subtype of malignant lymphoma that normally causes mucosal necrosis and destruction of nasal and sinus structures, which can make detection of lymphoma cells in necrotic tissue difficult, and this in turn made the differential diagnosis difficult. As shown in our case, endoscopic biopsy is more appropriate for diagnosis of FRS because it can more reliably exclude the presence of malignant disease. However, a smaller biopsy is normally preferred for diagnosis of both infectious and cancerous diseases to avoid dissemination of microorganisms and tumor cells in daily practice. Therefore, careful consideration is needed for the indication of a larger biopsy, and it should be noted that endoscopic sinus surgery was performed as therapy for intractable gangrene, as well as for diagnosis, in our case.

A second difficulty with diagnosis was that invasive FRS normally develops as an acute fulminant form in immunocompromised patients, especially those with DM. ${ }^{14}$ 


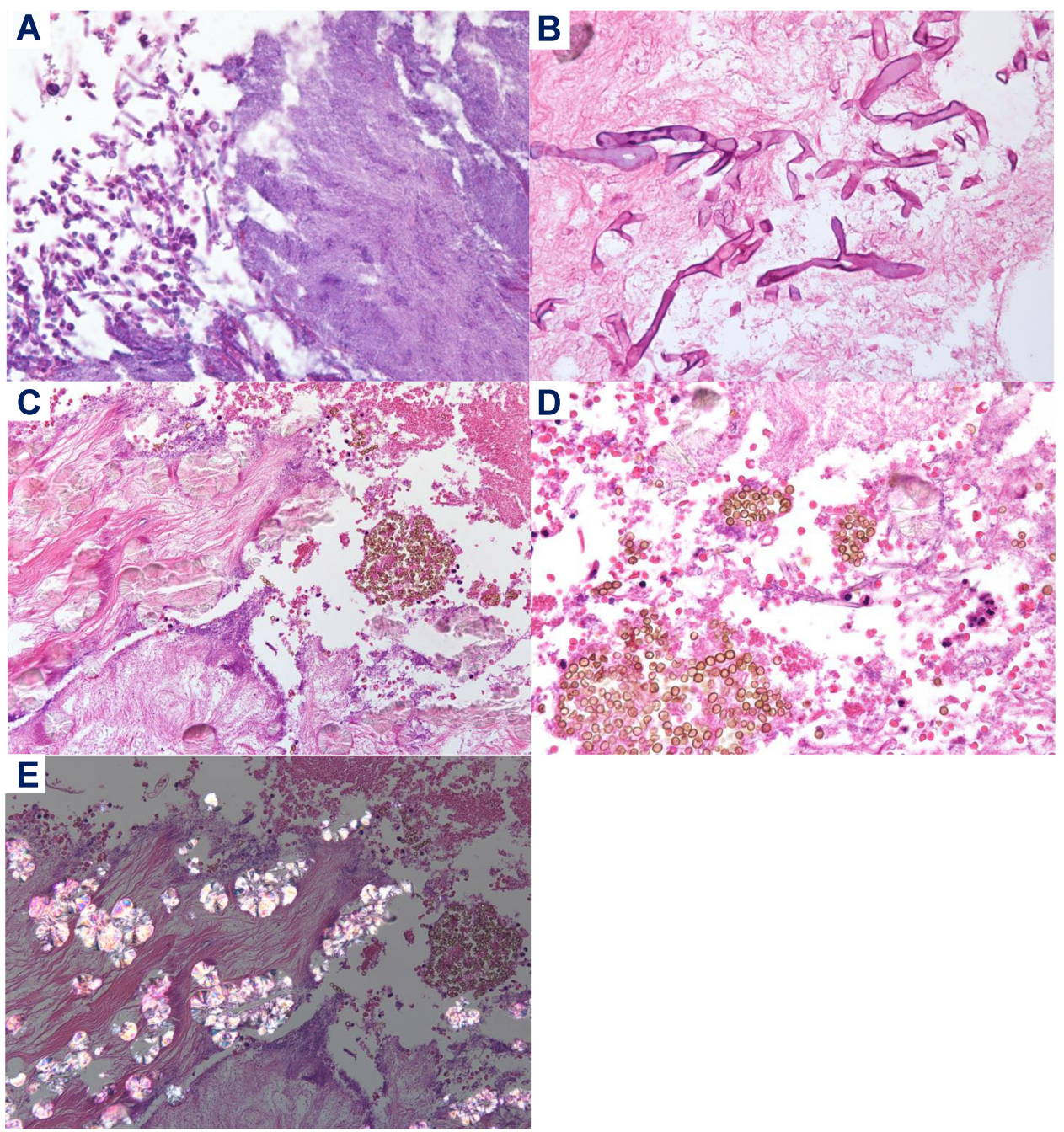

Figure 4 Histopathological findings. (A) Infiltrations of Candida species and Actinomycetes in the background of necrotic tissue of the soft palate (hematoxylin and eosin (HE) staining, $\times 600)$. (B) Infiltration of Mucor in the background of necrotic tissue of the soft palate (HE, $\times 400)$. (C-E) Low- $(\times 100)($ C) and high- $(\times 400)($ D) power magnification views of resected gangrene. Brown spores of Aspergillus niger and surrounding calcium oxalate crystals were identified in the background of necrotic tissue (E).

However, expansion of sinus gangrene took more than two months in our frail and immunocompromised patient with DM, who had recently had a failed renal transplantation and was dialysis-dependent. It is of note that invasive FRS due to a combination of Aspergillus and Mucor was the chronic form and did not progress to the intracranial region, but subtle differences in immunosuppressive status among patients may cause different disease progression patterns in invasive FRS. Actually, control of DM was relatively favorable on admission and immunosuppressants had already been discontinued before onset of CIFRS, but the patient was still frail and immunocompromised. This suggests the importance of improvement of underlying immunosuppressive disease, in addition to appropriate antifungal therapy, for successful treatment of invasive FRS. Finally, as also shown in our case, the prognosis of invasive FRS without orbit invasion has been found to be more favorable than that of FRS invading the orbit. ${ }^{19}$

In conclusion, we have reported an atypical case of CIFRS that was initially suspected to be ENKTL based on its unique progression pattern and laboratory data. This case suggests that CIFRS may occasionally occur in immunosuppressed patients and does not always progress towards the intracranial or orbital regions. In a case in which pathologic diagnosis is difficult with a small conventional biopsy specimen, large resection of gangrene can be both therapeutic and diagnostic for invasive FRS.

\section{Ethics and Consent Statement}

Informed consent has been provided by the patient for the publication of the case report and accompanying images. 


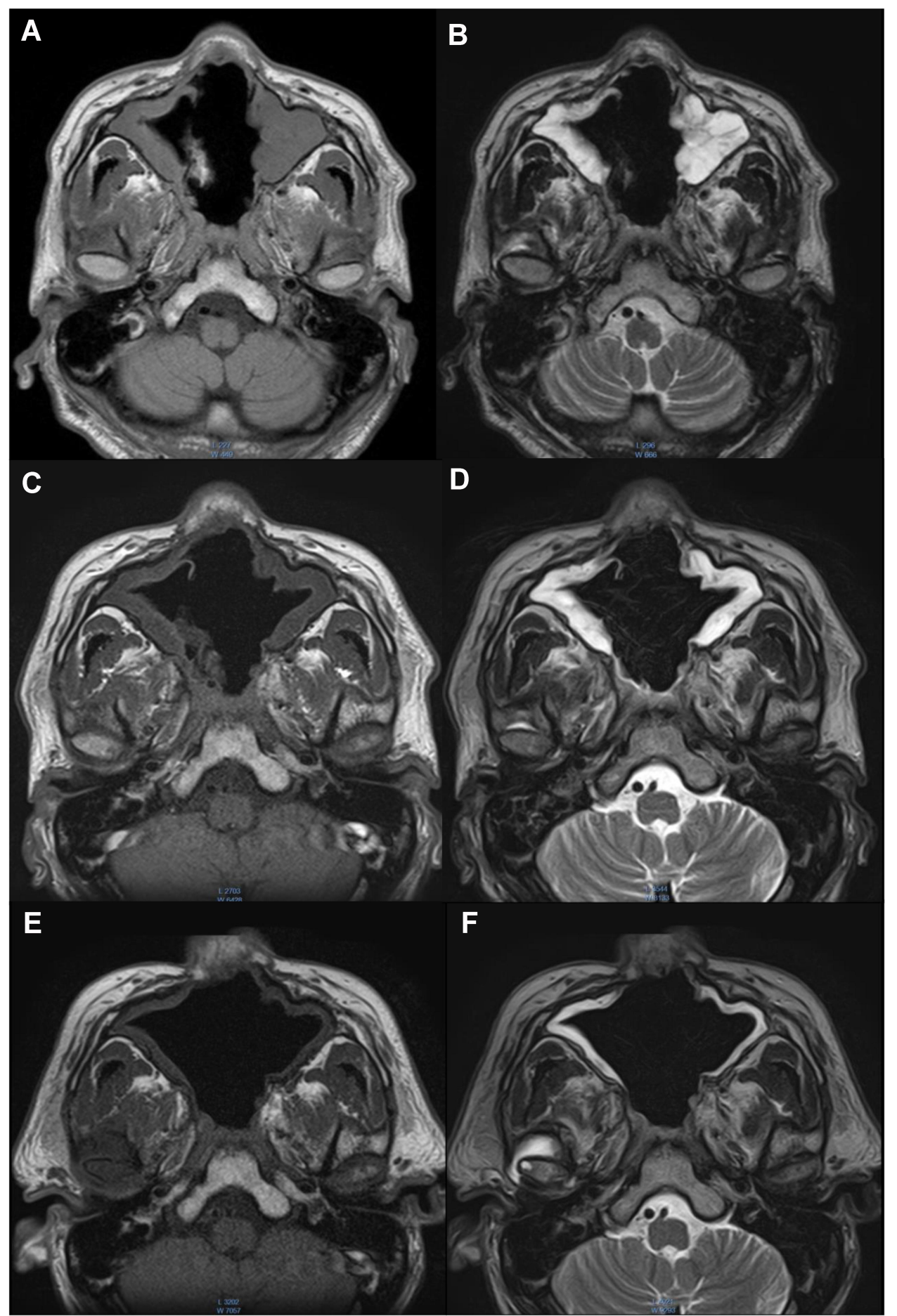

Figure 5 MRI findings along with clinical course. Horizontal views of ( $\mathbf{A}$ and $\mathbf{C}$ and $\mathbf{E})$ TI-weighted, and (B and $\mathbf{D}$ and $\mathbf{F})$ T2-weighted images at the level of the maxillary sinus after endoscopic sinus surgery ( $\mathbf{A}$ and $\mathbf{B}$ ), before the additional operation (C and $\mathbf{D})$, and five months after the additional operation (E and $\mathbf{F})$.

The institutional approval was not required for the publication of the case details.

\section{Disclosure}

Taku Tsukamoto has received research funding from Nippon Shinyaku. Tsutomu Kobayashi has received honoraria from
Chugai Pharmaceutical, Ono Pharmaceutical, Eisai, and Nippon Shinyaku. Junya Kuroda has received research funding from Celgene, Kyowa Kirin, Chugai Pharmaceutical, Ono Pharmaceutical, Sanofi, Eisai, Bristol-Myers Squibb, Sysmex, Astellas Pharma, Pfizer, Dainippon Sumitomo Pharma, Nippon Shinyaku, Takeda, Shionogi, Asahi Kasei, 
Daiichi Sankyo, MSD, Taiho Pharmaceutical, Fujimoto Pharmaceutical and Otsuka Pharmaceutical; honoraria from Janssen Pharmaceutical K.K, Celgene Corporation, Kyowa Kirin, Chugai Pharmaceutical, Ono Pharmaceutical, Takeda, Sanofi, Eisai, Bristol-Myers Squibb, Astellas Pharma, Pfizer, Nippon Shinyaku, Dainippon Sumitomo Pharma, Daiichi Sankyo, Fujimoto Pharmaceutical, Abbvie and Otsuka Pharmaceutical; and is a consultant for Janssen Pharmaceutical K.K, Celgene, Bristol-Myers Squibb, Sanofi and Abbvie. The authors report no other potential conflicts of interest for this work.

\section{References}

1. Chakrabarti A, Denning DW, Ferguson BJ, et al. Fungal rhinosinusitis: a categorization and definitional schema addressing current controversies. Laryngoscope. 2009;119(9):1809-1818. doi:10.1002/ lary. 20520

2. Deutsch PG, Whittaker J, Prasad S. Invasive and non-invasive fungal rhinosinusitis-a review and update of the evidence. Medicina (Kaunas). 2019;55(7):319. doi:10.3390/medicina55070319

3. Das A, Bal A, Chakrabarti A, et al. Spectrum of fungal rhinosinusitis; histopathologist's perspective. Histopathology. 2009;54(7):854-859. doi:10.1111/j.1365-2559.2009.03309.x

4. Montone KT, Livolsi VA, Feldman MD, et al. Fungal rhinosinusitis: a retrospective microbiologic and pathologic review of 400 patients at a single university medical center. Int $J$ Otolaryngol. 2012;2012:684835. doi:10.1155/2012/684835

5. Kimura M. Histopathological diagnosis of fungal sinusitis and variety of its etiologic fungus. Med Mycol J. 2017;58(4):J127-J132. doi:10.3314/mmj.17.018

6. Montone KT. Pathology of fungal rhinosinusitis: a review. Head Neck Pathol. 2016;10(1):40-46. doi:10.1007/s12105-016-0690-0

7. Singh AK, Gupta P, Verma N, et al. Fungal rhinosinusitis: microbiological and histopathological perspective. J Clin Diagn Res. 2017;11 (7):DC10-DC12.
8. deShazo RD, Chapin K, Swain RE. Fungal sinusitis. $N$ Engl J Med. 1997;337(4):254-259. doi:10.1056/NEJM199707243370407

9. Craig JR. Updates in management of acute invasive fungal rhinosinusitis. Curr Opin Otolaryngol Head Neck Surg. 2019;27 (1):29-36. doi:10.1097/MOO.0000000000000507

10. Pagella F, De Bernardi F, Dalla Gasperina D, et al. Invasive fungal rhinosinusitis in adult patients: our experience in diagnosis and management. $J$ Craniomaxillofac Surg. 2016;44(4):512-520. doi:10.1016/j.jcms.2015.12.016

11. Hora JF. Primary aspergillosis of the paranasal sinuses and associated areas. Laryngoscope. 1965;75:768-773. doi:10.1288/00005537196505000-00004

12. Afroze SN, Korlepara R, Rao GV, et al. Mucormycosis in a diabetic patient: a case report with an insight into its pathophysiology. Contemp Clin Dent. 2017;8(4):662-666. doi:10.4103/ccd. ccd_558_17

13. Ueno A, Yoneda M, Kimura Y, et al. An autopsied case of zygomycosis invasing in the central nervous system and vessels, which is difficult in the differential diagnosis from aspergillosis. Rinsho Shinkeigaku. 2012;52(2):84-89. doi:10.5692/clinicalneurol.52.84

14. Garas G, Choudhury N, Farrell R. Invasive fatal rhino-orbito-cerebral mucormycosis in diabetic ketoacidosis. JRSM Short Rep. 2010;1 (7):57. doi:10.1258/shorts.2010.010089

15. Taxy JB. Paranasal fungal sinusitis: contributions of histopathology to diagnosis: a report of 60 cases and literature review. Am J Surg Pathol. 2006;30(6):713-720. doi:10.1097/00000478-20060600000006

16. Li Z, Wang X, Jiang H, et al. Chronic invasive fungal rhinosinusitis vs sinonasal squamous cell carcinoma: the differentiating value of MRI. Eur Radiol. 2020;30(8):4466-4474. doi:10.1007/s00330-02006838-1

17. D'Andrea MR, Gill CM, Umphlett M, et al. Benefit of endoscopic surgery in the management of acute invasive skull base fungal rhinosinusitis. J Neurol Surg B. 2020.

18. Granville L, Chirala M, Cernoch P, et al. Fungal sinusitis: histologic spectrum and correlation with culture. Hum Pathol. 2004;35 (4):474-481. doi:10.1016/j.humpath.2003.10.024

19. Kasapoglu F, Coskun H, Ozmen OA, et al. Acute invasive fungal rhinosinusitis: evaluation of 26 patients treated with endonasal or open surgical procedures. Otolaryngol Head Neck Surg. 2010;143 (5):614-620. doi:10.1016/j.otohns.2010.08.017
Infection and Drug Resistance

\section{Publish your work in this journal}

Infection and Drug Resistance is an international, peer-reviewed openaccess journal that focuses on the optimal treatment of infection (bacterial, fungal and viral) and the development and institution of preventive strategies to minimize the development and spread of resistance. The journal is specifically concerned with the epidemiology of antibiotic resistance and the mechanisms of resistance development and diffusion in both hospitals and the community. The manuscript management system is completely online and includes a very quick and fair peerreview system, which is all easy to use. Visit http://www.dovepress.com/ testimonials.php to read real quotes from published authors. 